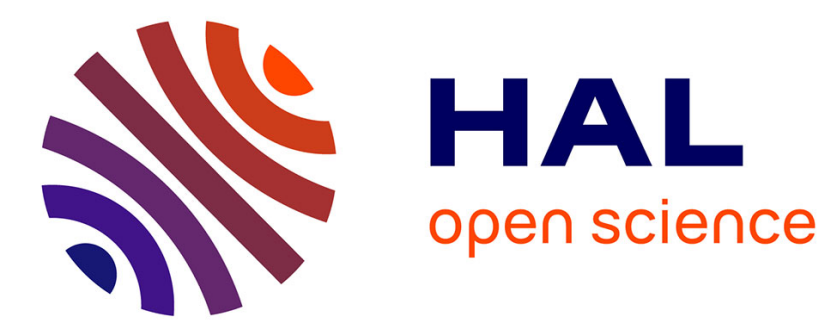

\title{
Optimal placement of drones for fast sensor energy replenishment using wireless power transfer
}

Christelle Caillouet, Tahiry Razafindralambo, Dimitrios Zorbas

\section{To cite this version:}

Christelle Caillouet, Tahiry Razafindralambo, Dimitrios Zorbas. Optimal placement of drones for fast sensor energy replenishment using wireless power transfer. WD 2019 - Wireless Days 2019, Apr 2019, Manchester, United Kingdom. 10.1109/WD.2019.8734203 . hal-02043123

\section{HAL Id: hal-02043123 \\ https://hal.inria.fr/hal-02043123}

Submitted on 20 Feb 2019

HAL is a multi-disciplinary open access archive for the deposit and dissemination of scientific research documents, whether they are published or not. The documents may come from teaching and research institutions in France or abroad, or from public or private research centers.
L'archive ouverte pluridisciplinaire HAL, est destinée au dépôt et à la diffusion de documents scientifiques de niveau recherche, publiés ou non, émanant des établissements d'enseignement et de recherche français ou étrangers, des laboratoires publics ou privés. 


\section{Optimal placement of drones for fast sensor energy replenishment using wireless power transfer}

\author{
Christelle Caillouet \\ Université Côte d'Azur, France \\ CNRS, I3S, Inria
}

\author{
Tahiry Razafindralambo \\ Université de La Réunion, France \\ LIM
}

\author{
Dimitrios Zorbas \\ Tyndall National Institute, Ireland \\ WSN group
}

\begin{abstract}
Lifetime is the main issue of wireless sensors networks. Since the nodes are often placed in inaccessible places, the replacement of their battery is not an easy task. Moreover, the node maintenance is a costly and time consuming operation when the nodes are high in numbers. Energy harvesting technologies have recently been developed to replenish part or all of the required energy that allows a node to function. In this paper, we use dedicated chargers carried by drones that can fly over the network and transmit energy to the nodes using radio-frequency (RF) signals. We formulate and optimally solve the Optimal Drone Placement and Planning Problem (OD3P) by using a given number of flying drones, in order to efficiently recharge wireless sensor nodes. Unlike other works in the literature, we assume that the drones can trade altitude with coverage and recharge power, while each drone can move across different positions in the network to extend coverage. We present a linear program as well as a fast heuristic algorithm to meet the minimum energy demands of the nodes in the shortest possible amount of time. Our simulation results show the effectiveness of our approaches for network scenarios with up to $\mathbf{5 0}$ sensors and a $50 \times 50 \mathrm{~m}$ terrain size.
\end{abstract}

\section{INTRODUCTION}

\section{A. Context}

Wireless sensor networks have brought a tremendous amount of civil, military and industrial applications in the context of the Internet of Things. They are capable of periodically monitoring their vicinity and therefore generating qualitative and quantitative measurements about their environment. Their main advantage is their low size and the ability to take measurements in real-time and forward them for further analysis. However, one of their main drawbacks is their limited battery lifetime.

Optimizing the battery consumption of wireless sensors has been the focus of many works in the literature. Since wireless sensors are autonomous objects powered by batteries, the performance of the underlying application strongly depends on their energy capacity. The data generation and transmission frequency of the sensors are always fundamental for the application. These two services consume the majority of the sensors' energy, thus, limiting the application's lifetime. Optimizing battery consumption from data generation and transmission frequency is important but not at the expense of the application.

Even though applications are already optimized, prolonging the lifetime of a wireless sensor network is still a fundamental issue. Since the deployment of the wireless sensors may

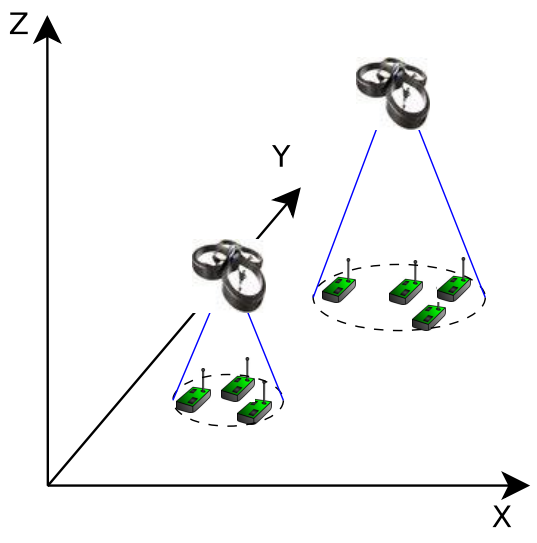

Fig. 1: An example employing 7 nodes and 2 drone positions.

happen in harsh environments where the sensors are not accessible, it is not always possible to replace the batteries with a low cost. To tackle or to alleviate this issue energy harvesting solutions have been proposed.

There are multiple energy harvesting solutions to increase the lifetime of sensor networks. On one hand, it is possible to harvest energy using, for example, solar panels or other types of harvesting. However, even if this type of harvesting is very effective and can greatly extend the sensors' lifetime, the amount of power is not constant and most of the time requires a big in size extra equipment. On the other hand, using a technology such as the RF-power harvesting [1], we can transmit a(n) (on-demand) constant amount of energy to the nodes, and recharge multiple nodes at the same time.

In this paper, we use the energy harvested by transmitted RF signals from flying drones to power up energy-constrained sensor nodes. We examine the scenario where the nodes are not always on, but we periodically visit them in order to (partially) recharge their battery and collect the data. We study the problem of optimally deploying flying drones over the sensor area. These flying drones carry dedicated chargers facing to the ground and are capable of emitting RF signals towards the sensors to recharge their battery as it is illustrated in the example of Fig.1. We develop a Mixed Integer Linear Program (MILP) and an heuristic to optimize drones utilization.

\section{B. Contributions}

Unlike previous works in the literature which do not consider realistic harvesting values neither drone mobility, (a) we 
take into account realistic harvesting values (for both transmitters and receivers) obtained from a RF-power harvesting module manufacturer, (b) we place the drones in 3D-space while taking into account the effect of the altitude adjustment to the harvesting power, and (c) we consider that the drones can change positions in the 3D-space to improve coverage and, thus, minimize the total energy replenishment time. To this end, we formulate and optimally solve the optimal drone placement and planning problem (OD3P) for the nodes energy replenishment using a mixed integer linear programming formulation. A sub-optimal greedy heuristic algorithm is also proposed as a time-efficient solution for large scale networks. We present extensive simulation results for network instances with up to 50 nodes and $50 \times 50 \mathrm{~m}$ terrain sizes and we study the effectiveness and scalability of the approaches.

\section{Outline}

The rest of this paper is organized as follows. Section II briefly reports the most recent related work. In Section III we describe in detail the OD3P problem. Then, in Section IV, we present the optimization model followed by an heuristic to solve OD3P in Section V. We then evaluate the performance of OD3P and we discuss its scalability in Section VI before concluding the paper in Section VII.

\section{RELATED WORK}

Recent works have already considered using vehicles as chargers to enhance wireless sensor network lifetime. However, they often limit the problem in a two-dimensional space [2] which decreases the accessibility and the recharge opportunities. Besides, some works focus only on a recharge model where the nodes are recharged one after the other [3]. Given the vehicle's lifetime restrictions, this solution may not be scalable since a considerable amount of time is spent to travel from one node to another. In our work, we can cover multiple nodes by a single drone depending on its position and the density of the network.

Optimal dedicated charger positioning problems are also examined in [4] and [5]. The first work presents a localized solution as well as centralized approximation algorithm to place mobile chargers among clustered wireless sensor nodes. The objective of the problem is to extend the lifespan of as many nodes as possible. An approximation algorithm for the same problem is presented in [5]. In [6], the potential of using battery-free nodes is examined. Dedicated chargers with fixed positions and omni-directional antennae are placed across the network to continuously transmit power to the nodes. A number of solutions for the charger placement problem while taking into account the electromagnetic radiation activity is, also, proposed in the literature [7], [8], [9]. However, all the previous works consider only 2-dimensional charger deployments and a non-realistic high-power omni-directional energy emission.

The financial cost of the deployment is an important parameter and [10] tries to minimize the number of drones used to maintain an operating wireless sensor network for a given amount of time. The authors provide a integer linear model related to the set cover problem and heuristics, to minimize the number of drones needed to wirelessly recharge the network. However, this work does not consider drone mobility and, thus, its practicality is limited only to very dense networks. In sparse networks, a number of drones that equals the number of nodes would be required.

\section{PRELIMINARIES}

Given a set of nodes $\mathcal{I}$ with known and fixed 2D positions, the goal is to optimally deploy and plan the positions of a set of drones $N$ in the 3D-space in order to provide an amount of energy at least equal to $E$ to all the nodes in the network in the shortest possible amount of time. This amount of energy is an empirical value that has to be sufficient to power-up a node, to allow it to take a measurement, and transmit the data to the drone (or to a base station). We assume that the transmission time is negligible compared to the recharge time of the nodes.

Each node is equipped with an RF-power harvesting module capable of converting the RF-power from transmitted signals to DC power. A charger with a directional antenna facing the ground is used to continuously emit power to the network and recharge the nodes. A drone can adjust its altitude to recharge multiple nodes at the same time.

The amount of power each node receives is affected by its distance to the transmission source (charger on the drone) and the environmental conditions. Formula (1) describes the power harvested by a node $i$ when it is located within the line of sight of the charger [11]:

$$
P_{h_{i}}=P_{r x}^{d_{i j}} f^{d_{i j}},
$$

where $P_{r x}^{d_{i j}}$ is the received power and $f^{d_{i j}}$ is the efficiency of the harvesting antenna at distance $d_{i j}$. The received power at distance $d$ is given by the following propagation model [12]:

$$
P_{r x}^{d}=P_{0} \frac{e^{2 \sigma G}}{d^{2 b}},
$$

where $\frac{e^{2 \sigma G}}{d^{2 b}}$ has a log-normal distribution with a shadowing coefficient $\sigma(G \sim N(0,1))$ and $b$ is the amplitude loss exponent. $P_{0}$ is the received power at reference distance.

The minimum harvested power received by a node to recharge its battery depends on the efficiency of the converter for the corresponding received power at this particular distance. We denote by $\Gamma$ this minimum harvesting threshold, which is a hardware-depended constant.

Formula (3) defines the harvested energy of a node $i$ for a given time period $t$ :

$$
H_{i}^{t}=\int_{0}^{t} P_{h_{i}} t d t .
$$

This energy is stored directly in a super-capacitor with some leakage properties expressed as $\eta H_{i}^{t}$, where $\eta \in(0,1)$.

\section{THE OD3P OPTIMIZATION MODEL}

We set $\mathcal{J}$ the set of possible drone 3D-positions and denote with $E_{i}$ the energy needs for each node $i$ in $\mathcal{I}$. We define 
as $\beta_{j}^{n}$ the binary variable stating whether a drone $n \in \mathcal{N}$ is deployed at position $j \in \mathcal{J}$. Let $\alpha_{n}$ be a binary variable stating if drone $n \in \mathcal{N}$ is used or not. We also add into the model the continuous variables $t_{j}^{n}$ representing the associated time a drone $n \in \mathcal{N}$ is located at position $j \in \mathcal{J}$. We finally set $\tau$ the maximum total recharge time of a drone. $\tau$ practically represents the drone's maximum flying time.

The goal is to minimize the time needed to harvest and store the required quantity of energy from the deployed drones to the sensors, while not exceeding the budget (in terms of number of drones) $B \leq|\mathcal{N}|$. We specify that at most one drone can be placed at each 3D-position, determining one possible altitude. The Mixed Integer Linear Program (MILP) is given as follows:

$$
\begin{aligned}
& \min \lambda \\
& \sum_{j \in \mathcal{J}} t_{j}^{n} \leq \lambda, \forall n \in \mathcal{N} \\
& \sum_{n \in \mathcal{N}} \sum_{j \in \mathcal{J}}(1-\eta) H_{i}^{t_{j}^{n}} \geq E_{i}, \forall i \in \mathcal{I} \\
& \sum_{n \in \mathcal{N}} \alpha_{n} \leq B \\
& \sum_{j \in \mathcal{J}} t_{j}^{n} \leq \tau \alpha_{n}, \forall n \in \mathcal{N} \\
& \sum_{n \in \mathcal{N}} \beta_{j}^{n} \leq 1, \forall j \in \mathcal{J} \\
& t_{j}^{n} \leq \tau \beta_{j}^{n}, \forall n \in \mathcal{N}, j \in \mathcal{J} \\
& \sum_{n \in \mathcal{N}} t_{j}^{n}+\sum_{j^{\prime} \in \mathcal{J},\left(x_{j}, y_{j}\right)=\left(x_{j^{\prime}}, y_{j^{\prime}}\right)}\left(\sum_{n^{\prime} \in \mathcal{N}} t_{j^{\prime}}^{n^{\prime}}\right) \leq \lambda, \forall j \in \mathcal{J} \\
& \alpha_{n} \in\{0,1\}, \beta_{j}^{n} \in\{0,1\}, t_{j}^{n} \in \mathbb{R}, \lambda \in \mathbb{R}
\end{aligned}
$$

The objective function seeks to minimize the longest drone flying time $\min \max _{n \in \mathcal{N}} \sum_{j \in \mathcal{J}} t_{j}^{n}$, which corresponds to the minimum recharge time that ensures the harvested energy needs per node. It has been decomposed into one objective (4) minimizing a continuous variable $\lambda$. This variable is, thus, the upper bound of the flying time of each drone (Constraints (5)).

Constraints (6) ensure that the harvesting energy is greater than the energy requirements of each sensor. To do so, we compute for each position $j \in \mathcal{J}$ the harvesting power for node $i \in \mathcal{I}$. We then verify that the sum over the positions harvesting power greater than $\Gamma$ to $i$ is enough to fulfill the node's requirement $E_{i}$. Constraint (7) ensures that the maximum budget is not exceeded. We can place each drone at different locations during the operation (Constraints (8)), but the total length of its flying time cannot exceed the imposed time limit (i.e., $\tau$ ). Only one drone can be placed at a given position (Constraints 9) in order to limit the movement of the drones through time. Constraints (11) verify that if a 2Dlocation with a corresponding altitude is chosen for a drone, then no other drone can be placed above or under it. In this way we avoid multiple drones to be aligned at different altitudes and, thus, limit physical collisions between the drones as well as interference.

OD3P problem is NP-hard since it is related to the set covering problem [10]. There is no algorithm that solves exactly OD3P in polynomial time. Due to the limited size of this paper, we omit a formal proof of hardness.

Solving the proposed model is challenging since there is no straightforward solution to determine the optimal recharge time while minimizing the drone's altitude. Indeed, placing the drones at low altitude the total recharge time can be improved since the harvesting power is increased. When we have enough available drones to recharge the sensors (i.e., Constraint (7) is not tight), the model will use them to reduce the average altitude of the selected positions. However, given the number of available drones, a trade-off exists here; by reducing the altitude of the drones, the coverage is limited and, thus, more successive drone positions are needed. On the contrary, due to lifetime limitations of the drones, the number of visiting positions may be also limited. This trade-off is captured by the model when computing the time associated to the position (i.e., the value of variable $t_{j}^{n}$ ). As far as more drones are utilized, the model will find the best trade-off between the number of positions visited by a drone (minimizing the sum of $t_{j}^{n}$ for all visited $j$ ), and the harvesting efficiency of each selected position (maximizing the sum of $H_{i}^{t_{j}^{n}}$ for all $j$ by selecting the appropriate altitude).

\section{OD3P GREEDY HEURISTIC ALGORITHM}

The complexity of MILP solution requires the use of a faster algorithm when dealing with topologies with a high number of nodes or drone positions. Hence, we propose a greedy heuristic algorithm to overcome this issue and approach the optimal solution.

The greedy algorithm (see Algorithm 1) computes a set $\mathcal{O}$ containing the set of sensors in decreasing order according to their energy needs $E_{i}$. The algorithm, then, selects the first encountered sensor in $\mathcal{O}$ that has the smallest set of possible harvesting 3D positions. This set is computed by comparing the received harvested power at node $i$ to threshold $\Gamma$. Every time a sensor is chosen, the 3D position with the highest harvesting power is selected. Given this position, the algorithm computes the maximum time length that the drone can spend at this position. We, thus, obtain a triplet $\left(i^{*}, j^{*}, t^{*}\right)$ composed of a sensor, a position, and an associated harvesting time.

If the position can be associated to a drone $n^{*}$, then the energy costs of the reached sensors from $j^{*}$ are updated and the position is marked as unavailable so that it cannot be further chosen. The sensors that are fully recharged are removed from the ordered set $\mathcal{O} . T_{j}$ is the available time for each position $j \in \mathcal{J}$. It depends on the time associated with same 2D-positions in order to ensure Constraints (11). If the cumulative flying time among same $2 \mathrm{D}$ positions reaches the time limit, then all these positions are removed so that they cannot be chosen. If no drone can go to position $j^{*}$ with associated time $t^{*}$ (i.e. if there is not enough time left), then position $j^{*}$ is simply discarded. $T_{n}$ is the available time for drone $n \in \mathcal{N}$. It is bounded by the maximum time period $\tau$, 
and seeks to be minimized. At the end of the first step of the algorithm, the flying time of the drone $n^{*}$ is updated. At the end of the algorithm, we compute the minimum value $\lambda$ of the recharging time phase.

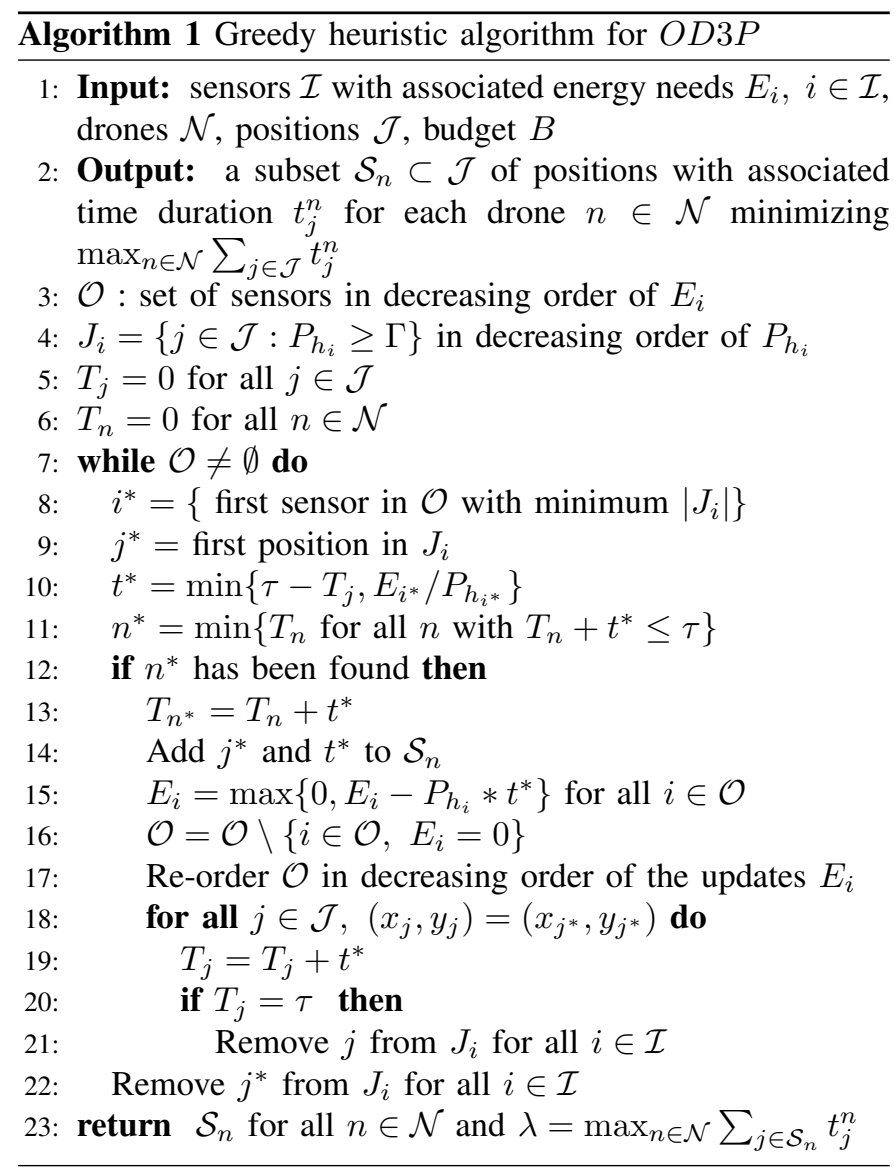

The computational complexity of the greedy algorithm depends on the number of nodes and the complexity of the implementation of the sorting algorithm $(O(n \log n)$ in best case). Since the algorithm seeks one node at each iteration (see line 7), and every iteration is computed in $O(m \log m)$ run time, where $m$ the number of nodes remaining in set $\mathcal{O}$, the total running time will be $\sum_{i=1}^{|I|} i \log i$.

\section{Performance eVAluation \& Discussion of the RESULTS}

\section{A. Simulation setup \& evaluation metrics}

We assume that the nodes are randomly placed on a 2dimensional grid of size $50 \mathrm{~m} \times 50 \mathrm{~m}$. We vary the number of nodes between 5 and 50, and the budget (in terms of number of drones) from 3 to 10 . The number of possible drone positions is chosen between 125 and 500 (i.e., $5 \times 5$ to $10 \times 10$ grid points $\times 5$ altitudes). The energy requirement of each sensor is set to $150 \mathrm{~mJ}$. For instance, this is the minimum amount of energy a Zolertia Z1 node ${ }^{1}$ needs in order to bootup, take a measurement, and transmit it to the gateway. The

\footnotetext{
${ }^{1}$ https://github.com/Zolertia/Resources/wiki/The-Z1-mote
}

drones can cover the nodes' energy demands by transmitting $3 \mathrm{~W}$ EIRP power $(915 \mathrm{MHz})$ to the nodes, which according to our adopted path-loss model and the manufacturer's data results in approximately $\left[\Gamma=6.3 \times 10^{-5}, 0.01\right] \mathrm{W}$ of harvested power depending on the distance. The power harvesting values are computed given the efficiency of the Powercast $\mathrm{P} 2110 \mathrm{~B}$ modules $^{2}$ operating at $915 \mathrm{MHz}$. The efficiency varies from 0 to about $65 \%$ depending on the received power. The chosen parameters for the harvesting model are $P_{0}=10 \mathrm{~mW}$, $b=1.05, \sigma=1$, and $\eta=0.3$. The maximum recharging time of a drone is set to 1 hour.

For each size of $\mathcal{I}, \mathcal{J}$ and budget $B \leq|\mathcal{N}|$, we compute 10 different random topologies. We summarize the results with the mean value for each topology size. We did not plot the 95\% confidence interval to keep the plot readable since they are very close to the mean value. The model is implemented in Java language and solved using the IBM CPLEX solver 12.8 on an Intel Core i7-5500U CPU, $2.40 \mathrm{GHz}, 16 \mathrm{~Gb}$ RAM computer under Microsoft Windows 10 operating system.

\section{B. Study of the optimal energy replenishment time}

In this study we compute the optimal time needed to replenish the energy of the sensors for different numbers of used drones, sensors, and 3D-positions (grid positions). The results are computed by solving the OD3P optimization problem and are depicted in Fig. 2. They reveal the model's ability to compute optimal values even for a high number of sensors and drone positions. On one hand, as it was expected, the recharge time decreases as more drones are added in the field or more drone positions are taken into account. More drones are also needed for higher node populations. Moreover, the optimal number of drones varies between 7 and 9 drones. On the other hand, the recharge time tends to remain constant or increase as more drones are added above the optimal point. This happens because once the optimal solution has been found, the model does not allow placing drones at different altitudes while covering the same number of sensors. As a consequence, some drones are slightly moved from the optimal position so that the rest of the drones can fit in the 3D field. This results in a constant or sometimes higher recharge time.

\section{Study of the drone positions}

In the next study, we compute the number of successive positions of the drones throughout the process as well as the mean recharge time per position. We remind that each drone is allowed to change positions in order to recharge as many sensors as possible within the imposed time limit. As we can observe from Fig.3a, a drone moves on average to several positions in order to fulfill the energy requirements of the nodes (Constraints (6)). More positions are selected as more nodes are added in the field. Moreover, the number of positions decreases when increasing the drone population. Despite that, it is interesting to observe that the number of positions converges to 2 or 3 . This number is related to the

\footnotetext{
${ }^{2} \mathrm{http}: / /$ www.powercastco.com/wp-content/uploads/2016/12/P2110BDatasheet-Rev-3.pdf
} 


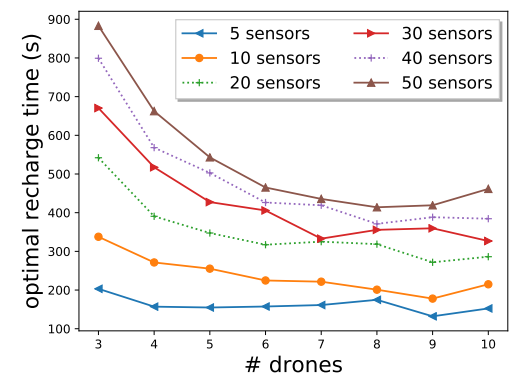

(a) Variable number of drones \& sensors.

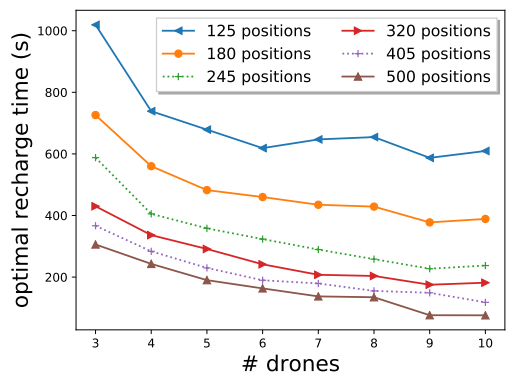

(b) Variable drone positions \& populations.

Fig. 2: Optimal energy replenishment time for (a) variable drone and sensor populations, and (b) variable drone positions and drone populations.

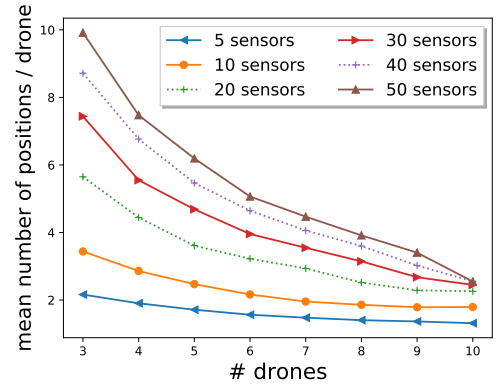

(a) Number of successive positions.

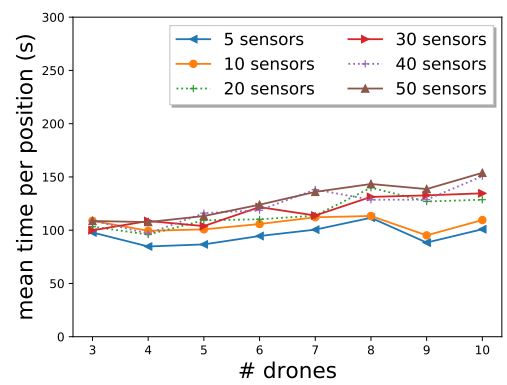

(b) Mean recharge time per position.

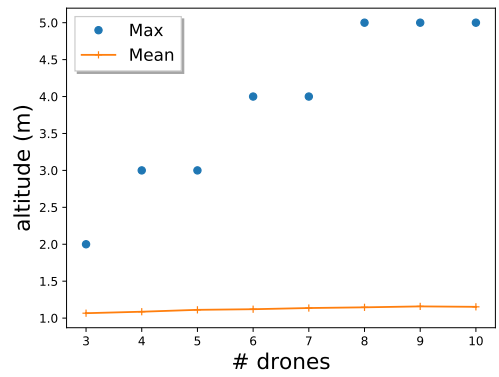

(c) Maximum and mean altitudes.

Fig. 3: Analysis of the computed positions per drone \& time per position.

minimum time required to recharge a single node at the lowest altitude as well as to the imposed energy replenishment time. This can be also explained by dividing the results of Fig.2a by those of $3 b$, which will give us the mean number of drone positions.

Placing the drones at the lowest possible altitude, the harvesting energy is maximized due to the increased power and the high efficiency of the harvesting module. However, this action decreases coverage, thus, more drones are needed in the field. In other words, as explained in Section IV there is a trade-off between coverage and recharging time that the computational model has to solve. Fig.3c reflects the behavior of the model while dealing with this trade-off. The figure reports the mean and the maximum altitude of the drones for the examined scenarios. The results witness that even if there are selected positions at $3 m$ (for $|\mathcal{J}|=125$ or 250 ), or $5 m$ (for bigger $|\mathcal{J}|$ ), the average altitude of all the selected positions barely exceeds $1 \mathrm{~m}$. This shows that low altitude positions are promoted by the model.

\section{Scalability study \& comparison}

In this study we examine the computational model's scalability and we compare MILP's performance to that of the greedy algorithm.

Fig. $4 \mathrm{~b}$ presents the execution time of MILP for different drone positions and populations. The MILP optimally solves all the examined instances. However, the resolution time presents an exponential increase as the number of drone positions and the number of drones increases. However, as depicted in Fig. 4a and 4b, it needs only a few seconds for scenarios with up to 20 nodes and up to 245 drone positions, respectively. On the contrary, the greedy algorithm solves quasi instantly all the topologies. Indeed, the maximum captured running time of the algorithm is $72 \mathrm{~ms}$. We must note that, in terms of total recharge time, the greedy approach achieves the optimal value for only $8.2 \%$ of the instances, while about $17 \%$ of the solutions are up to $10 \%$ longer than the optimal. The optimal value is usually reached for small number of drone positions (i.e., 125, 180, and 245). When the problem complexity increases greedy is on average $26.07 \%$ far from the optimal (Fig. 5a). Moreover, as we can observe from Fig. $5 \mathrm{~b}$ and $5 \mathrm{c}$, the solutions computed by the heuristic impose on average a lower drone altitude as well as a lower recharge time per position. This result reveals the ability of the model to provide a better trade-off between the altitude and the optimal recharge time compared to the heuristic algorithm.

\section{CONCLUSION AND FUTURE WORK}

In this paper, we presented an optimal linear formulation of the drone placement and planning problem in order to minimize the energy replenishment time of ground sensors. We developed and optimally solved a Mixed Integer Linear Program (MILP) to tackle the problem. We also proposed a heuristic algorithm capable of solving large instances of the problem in a very short amount of time. The originality of our work lies on the focus of our approach which is the 


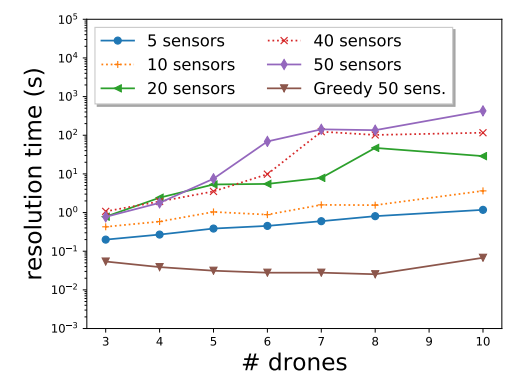

(a) Variable number of sensors.

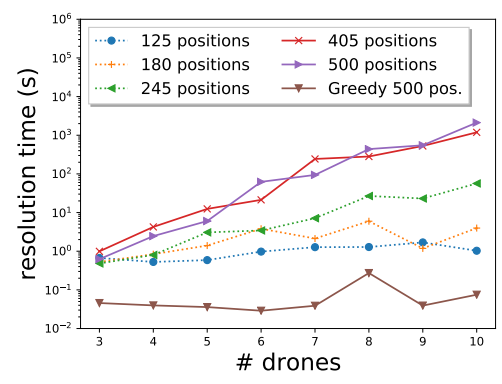

(b) Variable number of drone positions.

Fig. 4: Resolution time for variable number of nodes and drone positions.

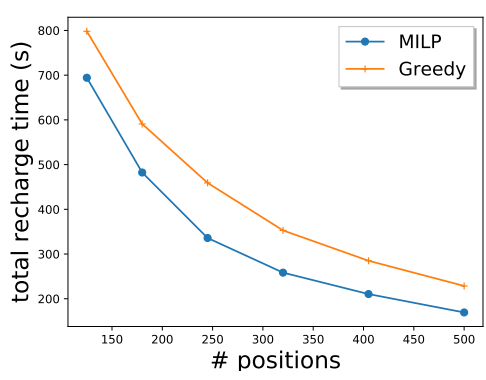

(a) Total recharge time.

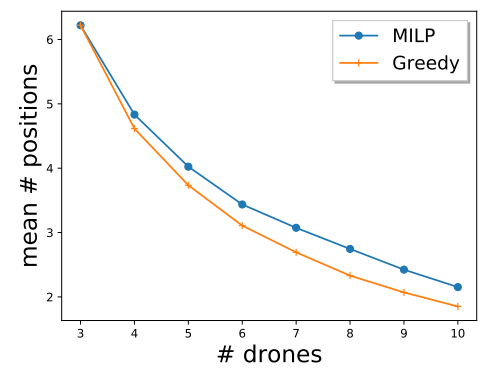

(b) Mean number of successive drone positions.

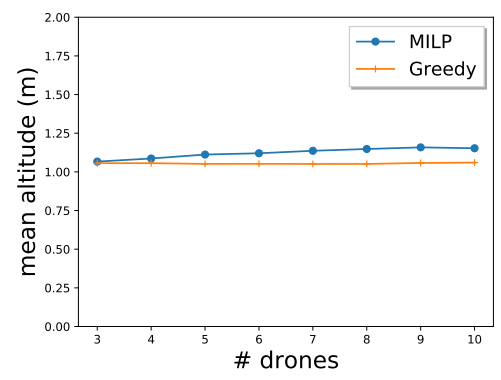

(c) Mean drone altitude.

Fig. 5: Comparison with the greedy algorithm.

minimization of the recharging time, given a number of mobile drones. The simulation results showed the effectiveness of our strategy both in terms of deployment and computation cost. They also showed that the drones altitude is minimized to ensure good harvesting capabilities which gives good insights to the analysis of practical distributed algorithms and industrial scenarios. In the next steps of this work, we would like to investigate a fully localized algorithm for the drone placement and planning problem. We will also focus on integrating the drones' trajectories inside the MILP and the heuristic formulation.

\section{ACKNOWLEDGMENTS}

This publication has emanated from research conducted with the financial support of Science Foundation Ireland (SFI) and is co-funded under the European Regional Development Fund under Grant Number 13/RC/2077.

It has also received funding from the European Union's Horizon 2020 research and innovation program under the Marie Skodowska-Curie grant agreement No. 713567, and by the ANR program Investments for the Future under reference ANR-11-LABX-0031-01.

\section{REFERENCES}

[1] X. Lu, P. Wang, D. Niyato, D. Kim, and Z. Han, "Wireless networks with rf energy harvesting: A contemporary survey," IEEE Comm. Surveys and Tutorials, vol. 17, no. 2, pp. 757-789, 2015.

[2] S. Zhang, Z. Qian, F. Kong, J. Wu, and S. Lu, "P3: Joint optimization of charger placement and power allocation for wireless power transfer," in IEEE Infocom, April 2015, pp. 2344-2352.
[3] N. Najeeb and C. Detweiler, "Extending wireless rechargeable sensor network life without full knowledge," in Sensors, vol. 17, no. 7, 2017.

[4] D. Zorbas, P. Raveneau, and Y. Ghamri-Doudane, "On optimal charger positioning in clustered rf-power harvesting wireless sensor networks," in Proceedings of the 19th ACM International Conference on Modeling, Analysis and Simulation of Wireless and Mobile Systems, ser. MSWiM '16. New York, NY, USA: ACM, 2016, pp. 225-228.

[5] Y. Pang, Z. Lu, M. Pan, and W. W. Li, "Charging coverage for energy replenishment in wireless sensor networks," in Proceedings of the 11th IEEE International Conference on Networking, Sensing and Control, April 2014, pp. 251-254.

[6] D. Zorbas, P. Raveneau, Y. Ghamri-Doudane, and C. Douligeris, "On the optimal number of chargers in battery-less wirelessly powered sensor networks," in 2017 IEEE Symposium on Computers and Communications (ISCC), July 2017, pp. 1312-1317.

[7] H. Dai, Y. Liu, A. X. Liu, L. Kong, G. Chen, and T. He, "Radiation constrained wireless charger placement," in IEEE INFOCOM 2016 - The 35th Annual IEEE International Conference on Computer Communications, April 2016, pp. 1-9.

[8] Y. Wang, W. Yang, X. Shang, J. Hu, Y. Huang, and Y. Cai, "Energyefficient secure transmission for wireless powered internet of things with multiple power beacons," IEEE Access, pp. 1-1, 2018.

[9] T. Rault, "Avoiding radiation of on-demand multi-node energy charging with multiple mobile chargers," Computer Communications, vol. 134, pp. $42-51,2019$.

[10] D. Zorbas and C. Douligeris, "Computing optimal drone positions to wirelessly recharge iot devices," in The 11th International Workshop on Wireless Sensor, Robot and UAV Networks (WiSARN), Apr. 2018.

[11] D. Zorbas, P. Raveneau, and Y. Ghamri-Doudane, "Assessing the cost of rf-power harvesting nodes in wireless sensor networks," in 2016 IEEE Global Communications Conference (GLOBECOM), Washington, DC, USA, Dec 2016.

[12] M. Z. Win, P. C. Pinto, and L. A. Shepp, "A mathematical theory of network interference and its applications," Proceedings of the IEEE, vol. 97, no. 2, pp. 205-230, Feb 2009. 